\title{
An Integrated Hybrid Transportation Architecture for Human Mars Expeditions
}

\author{
Raymond G. Merrill ${ }^{1}$, Patrick R. Chai ${ }^{2}$ \\ NASA Langley Research Center, Hampton VA 23681 \\ and \\ $\operatorname{Min} \mathrm{Qu}^{3}$ \\ Analytical Mechanics Associates, Inc., Hampton, VA 23666
}

\begin{abstract}
NASA's Human Spaceflight Architecture Team is developing a reusable hybrid transportation architecture that uses both chemical and electric propulsion systems on the same vehicle to send crew and cargo to Mars destinations such as Phobos, Deimos, the surface of Mars, and other orbits around Mars. By applying chemical and electrical propulsion where each is most effective, the hybrid architecture enables a series of Mars trajectories that are more fuel-efficient than an all chemical architecture without significant increases in flight times. This paper presents an integrated Hybrid in-space transportation architecture for piloted missions and delivery of cargo. A concept for a Mars campaign including orbital and Mars surface missions is described in detail including a system concept of operations and conceptual design. Specific constraints, margin, and pinch points are identified for the architecture and opportunities for critical path commercial and international collaboration are discussed.
\end{abstract}

$\begin{array}{ll} & \\ \text { BLT } & =\text { Ballistic Lunar Transfer } \\ \text { CP } & =\text { Chemical Propulsion } \\ \text { COMPASS } & =\text { Collaborative Modeling for Parametric Assessment of Space Systems } \\ \Delta \mathrm{V} & =\text { Change in velocity } \\ \text { ECLSS } & =\text { Environment Control and Life Support Systems } \\ \text { EM } & =\text { Exploration Mission } \\ \text { EMC } & =\text { Evolvable Mars Campaign } \\ \text { EP } & =\text { Electric Propulsion } \\ \text { EUS } & =\text { Exploration Upper Stage } \\ \text { GRC } & =\text { Glenn Research Center } \\ \text { HAT } & =\text { Human Spacefligth Archtiecture Team } \\ \mathrm{I}_{\text {sp }} & =\text { Specific Impulse } \\ \text { ISS } & =\text { International Space Station } \\ \text { KSC } & =\text { Kennedy Space Center } \\ \text { L1 } & =\text { Earth }- \text { Moon Lagrange Point } 1 \\ \text { L2 } & =\text { Earth }- \text { Moon Lagrange Point } 2 \\ \text { LDHEO } & =\text { Lunar Distance High Earth Orbit } \\ \text { LDRO } & =\text { Lunar Distant Retrograde Orbit } \\ \text { LEO } & =\text { Low Earth Orbit } \\ \text { LGA } & =\text { Lunar Gravity Assist } \\ \text { LNRO } & =\text { Lunar Near Rectilinear Orbit } \\ \text { MMH } & =\text { Monomethylhydrazine }\end{array}$

${ }^{1}$ Aerospace Engineer, Space Mission Analysis Branch, MS 462, AIAA Senior Member.

${ }^{2}$ Aerospace Engineer, Space Mission Analysis Branch, MS 462, AIAA Member.

${ }^{3}$ Staff Scientist, 21 Enterprise Parkway, Suite 300, Hampton, VA 23666-6413. 
NASA $=$ National Aeronautics and Space Administration

NTO $=$ Nitrogen Tetroxide

PEV $\quad=$ Pressurized Excursion Vehicle

RCS $=$ Reaction Control System

SEP $\quad=$ Solar Electric Propulsion

SLS $\quad=$ Space Launch System

$\mathrm{V}_{\infty} \quad=$ Excess Hyperbolic Velocity

\section{Introduction}

$\mathrm{T}$ HE National Aeronautics and Space Administration (NASA) is currently developing options for potential future paths as part of an Evolvable Mars Campaign (EMC) that expands human presence from Low Earth Orbit (LEO) into the solar system and to the surface of Mars. The strategic principles set forth for the $\mathrm{EMC}^{1}$ dictate that the journey to Mars involves an incremental buildup of capabilities while fielding interesting and exciting human space missions at a defined cadence without an increase to current funding levels. The systems built must be part of an evolvable multi-use infrastructure that provides significant opportunities for commercial and international involvement based on International Space Station (ISS) agreements and capabilities. These campaigns begin with Earth reliant missions to expand the knowledge of operations in space, continue with missions in cis-lunar space for testing and certification of required technologies, and ultimately result in Earth independent missions and long duration stays on the Martian surface. For any Mars exploration mission, the round trip transportation of systems from Earth to Mars remains a challenging problem that is strongly coupled with both deployment in Earth's sphere of influence and operations in Mars sphere of influence. In-space transportation architectures from historical NASA Mars studies include concepts that utilize portfolios of new technologies including long duration zero boil-off cryogenic storage, zero leak cryogenic valves, nuclear thermal propulsion, and megawatt class electric propulsion. ${ }^{2,3}$ By requiring such a portfolio of new technologies, a significant increase in budget is required to field even an initial mission. Typically missions only occur once all components are ready to support a Mars surface mission including entry descent landing (EDL), ascent, and surface systems. In previous studies in-space transportation elements are disposed of after a single use; although recapture is occasionally mentioned, fuel for recapture, repair-ability, refitting, and reuse are not considered or designed into the system. ${ }^{4}$ The Hybrid in-space transportation architecture ${ }^{5}$ is being developed to enable reuse of in-space transportation stages. An integrated campaign based on the Hybrid that includes a Phobos orbital expedition and two Mars surface expeditions is presented in this paper. It includes reuse and refueling of the in-space transportation vehicles and is one option for a set of Mars missions that are under development by the Human Spaceflight Architecture Team (HAT) as an attempt to satisfy the EMC strategic goals for missions that occur after cis-lunar proving ground missions. The specific focus to date has been on development of the in-space transportation system as one potential pathway to Mars for consideration.

\section{Hybrid in-Space Transportation Architecture and Trajectories}

The Hybrid in-space transportation architecture is being developed specifically to enable reusable transportation stages. Additional implementation goals to determine feasibility of an all-up round-trip reusable transportation capability for crew and cargo transit to Mars are:

1) No pre-deployment of return assets at destination, and

2) Pre-integrated propulsion and habitation systems prior to launch, and

3) Return of entire spacecraft to cis-lunar space, and

4) Lower electric propulsion (EP) power than previous studies, $<300 \mathrm{~kW}$ if possible, and

5) Use existing or in-development capabilities, and

6) $\geq 300$ day Mars orbit dwell time, and

7) < 1100 day round trip heliocentric mission duration, and

8) Use identical propulsion module for crew missions and cargo missions.

These goals are achieved by using the most efficient staging points, trajectories, and propulsion systems possible while balancing time-of-flight for deployment, aggregation, and transit. Maximum orbital energy for the transportation system is maintained at Earth by using the part of cis-lunar space with low energy with respect to the Moon as a "Basecamp" for preparing and resupply after completion of a mission beyond Earth. Low energy cislunar space includes families of orbits such as lunar distant retrograde orbits (LDRO), L1 and L2 Halo orbits, lunar near rectilinear orbits (LNRO), and other quasi stable orbits at the boundary of the Earth and the Moon's gravity well. 
Both cargo and crew are launched to low energy cis-lunar space through the part of cis-lunar space with high energy with respect to the Moon. For crew, a fast transfer with powered lunar gravity assists (LGA) is used with durations that can be characterized as a "taxi" class transit of 8-10 days, while cargo can be deployed on slower ballistic lunar transfers (BLT) ${ }^{7}$ that take $120-200$ days to maximize cargo delivery capability. These two types of transits between low energy cis-lunar and lunar distance high Earth orbit (LDHEO) can also used to prepare for Earth departure, with the quick transit costing $300-400 \mathrm{~m} / \mathrm{s}$ change in velocity $(\Delta \mathrm{V})$ and the slow transit costing $0-$ $40 \mathrm{~m} / \mathrm{s} \Delta \mathrm{V}$.

At Mars, a large elliptical orbit, with a period of 5 Martian days, or 5-sol, is used to maintain orbital energy of the transit stage while still enabling less than 3 day "taxi" class transits transit to and from either Phobos, Deimos, or the Martian surface. The energy required to transfer from Earth to Mars and back is set by planetary alignment, and past studies ${ }^{8}$ have identified the lowest energy direct routes between the planets for given round trip time-of-flight and stay time. The Hybrid architecture uses trajectories that can be considered direct minimum energy conjunction class and are constrained to a maximum heliocentric time of flight of 1100 days with at least 300 days in Martian sphere of influence. The approximately one year duration for transit allows for use of solar electric propulsion (SEP) to make up the difference in energy required between departure and arrival for either inbound or outbound transits. Use of SEP at power levels less than one third of previous concepts for Mars-piloted missions enables significant reduction in required arrival and departure chemical $\Delta \mathrm{V}$ while taking advantage of an order of magnitude increase in efficiency, in terms of specific impulse $\left(\mathrm{I}_{\mathrm{sp}}\right)$, of the propulsion system. At Earth a series of LGA can be used to either depart or capture into LDHEO or a BLT from a hyperbolic excess velocity $\left(\mathrm{V}_{\infty}\right)$ of $\geq 1.4 \mathrm{~km} / \mathrm{s}$ without expending propellant. ${ }^{9,10}$ At Mars a small burn of the Hybrid main propulsion system provides the transition from heliocentric space with a $V_{\infty}$ of $1-1.5 \mathrm{~km} / \mathrm{s}$ to the 5 -sol parking orbit. This same chemical propulsion system is also

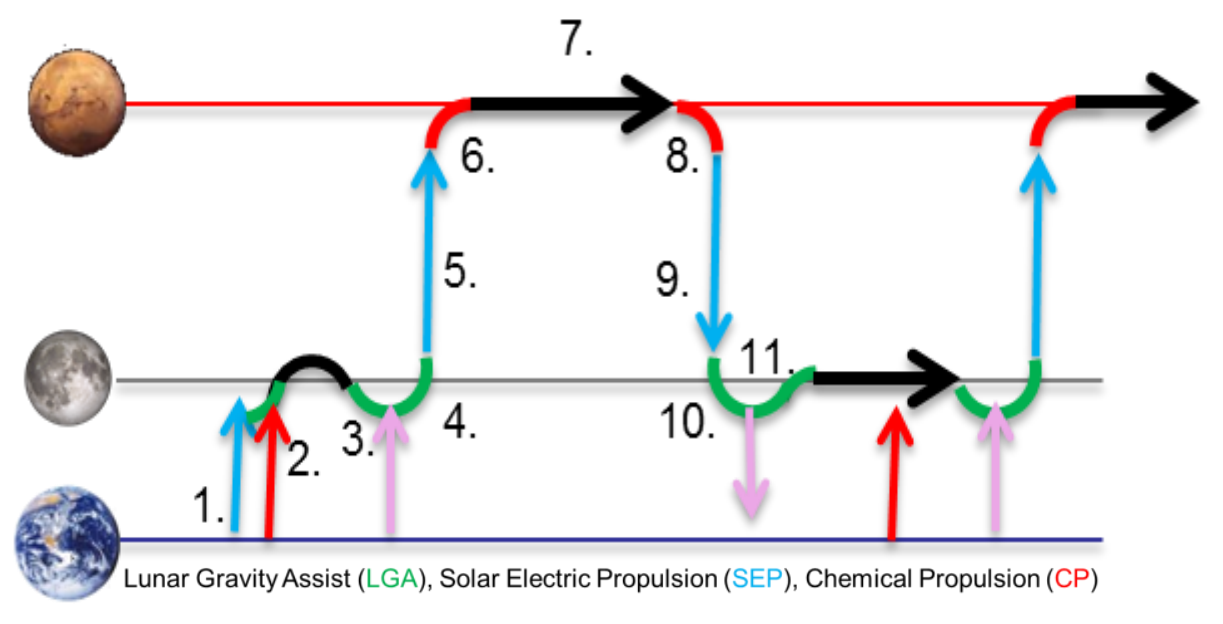

1) Deploy to cis-lunar space

2) Refuel and outfit

3) Crew and final logistics rendezvous

4) LGA series for Earth departure

5) Solar Electric Propulsion (SEP) thrusting to Mars

6) Chemical Propulsion (CP) burn at Mars close approach to insert into 5-sol parking orbit

7) Mars destination operations and realignment

8) $\mathrm{CP}$ burn at periapsis for Mars departure

9) SEP thrusting to Earth

10) LGA series for Earth capture into cis-lunar space

11) Crew return to Earth surface

Repeat steps 2-11 for each piloted Mars mission

Figure 1. Hybrid Architecture Mission Phases 
used to reorient the transit vehicle at Mars from the arrival to departure parking orbit, in some cases with the aid of SEP thrusting for slow reorientations. Specific details on trajectory designs for the Hybrid architecture are documented by Qu. ${ }^{11}$ Figure 1 is a chronological Hybrid mission to Mars for crew. Cargo missions utilized in this instantiation of the architecture are similar, with steps 3 and 10 unnecessary. Earth departure and arrival can be from a BLT without the stop-over in LDHEO required for crew rendezvous and departure. Stop-over, however, is possible for rendezvous with cargo immediately prior to Earth departure, increasing both the flexibility of launch scheduling and the robustness of the manifest to launch delays.

\section{Hybrid Spacecraft, Refueling Tanker and EMC Flight Elements}

The HAT is developing a set of spacecraft concepts to be used in construction of potential paths on the road to Mars. In early 2015 more detailed options concpets for the Hybrid spacecraft and refueling tankers were developed in order to prove performance viability of the Hybrid architecture. Other flight elements presented are common across architectures for HAT and are based roughly on Mars DRA 5.0 architectural assumptions, ${ }^{12}$ except with regard to the larger 5-sol piloted transfer vehicle Mars parking orbit.

\section{A. Hybrid Propulsion Stage}

The Hybrid Propulsion Stage (HPS) is the heart of the Hybrid architecture. Initially a high level parametric equation for spacecraft mass with respect to four key parameters -EP power, xenon load, hypergolic biprop. fuel load, and total array power- was developed and used to show the potential feasibility of a Hybrid Architecture. ${ }^{5}$ That relationship was used to determine the range of key parameters for a given payload mass as a starting point for more detailed concept development. The Glenn Research Center COMPASS ${ }^{13}$ team, in collaboration with the authors, developed two concepts for a Hybrid Propulsion Stage (HPS) shown in Figure 2. The concepts are designed with currently available components where possible and are limited to supporting three missions to Mars, with element lifetime of approximately 15 years.

The SEP system uses components in development for the the Asteroid Redirect Mission (ARM) SEP stage.

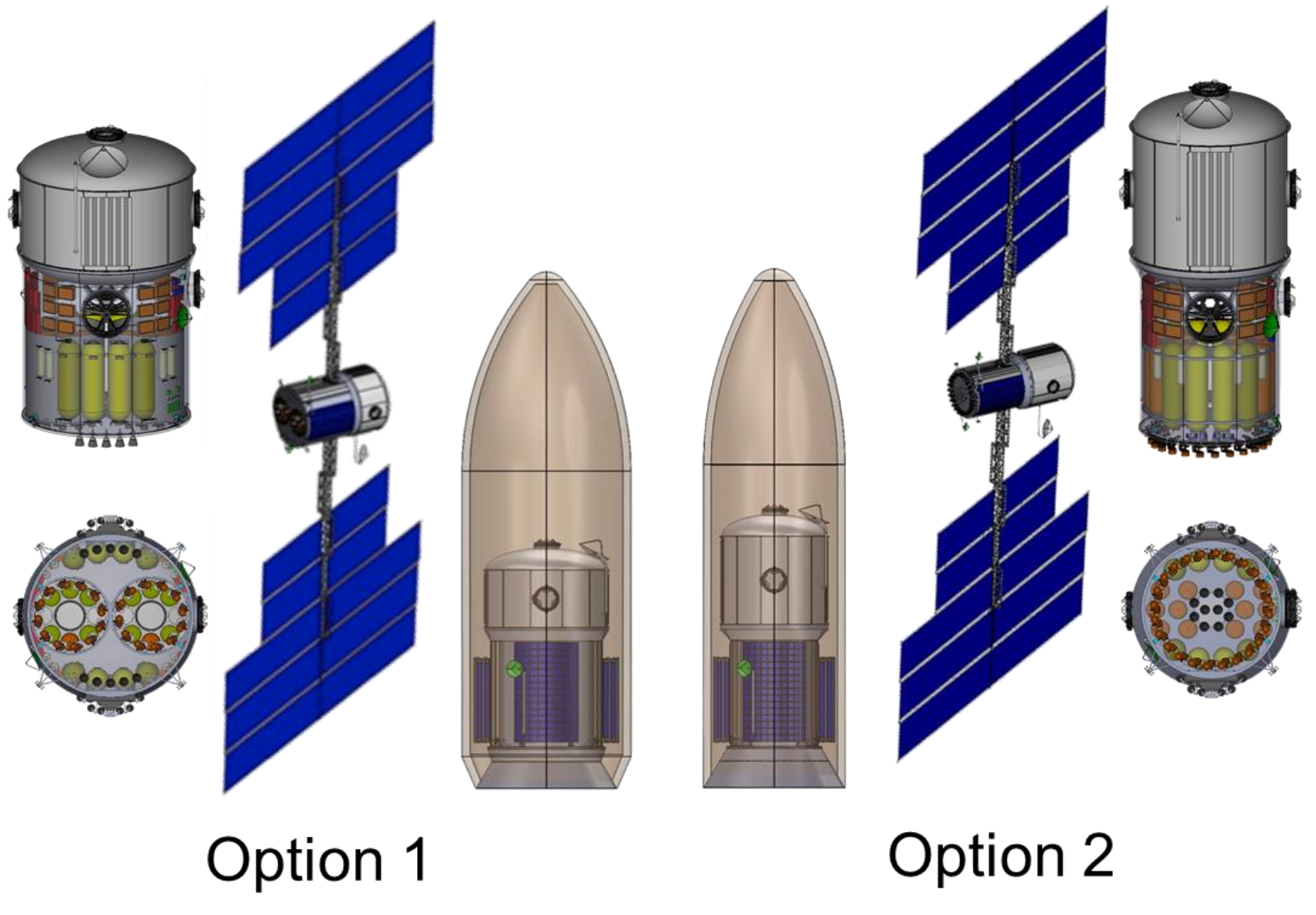

Figure 2. GRC COMPASS Hybrid Propulsion Stage Options

American Institute of Aeronautics and Astronautics 
Initial investigation into the possibility of incorporating two identical $150 \mathrm{~kW}$ Block $1 \mathrm{~A}^{14}$ evolved ARM spacecraft buses provides a basis for the total EP system input power of approximately $318 \mathrm{~kW}$. It is composed of $24 \mathrm{EP}$ strings, each composed of a power processing unit (PPU), xenon feed system, and Hall Effect Rocket with Magnetic Shielding (HERMeS) thruster ${ }^{15}$ that can operate in high $\mathrm{I}_{\mathrm{sp}}(\sim 3000 \mathrm{~s})$ or low $\mathrm{I}_{\mathrm{sp}}(\sim 2000 \mathrm{~s})$ modes. The option 1 Hybrid concept is the result of attempting to use the ARM block 1A spacecraft bus SEP module as designed. During the initial detailed design activity for the EP system it became clear that the identical ARM spacecraft could not be used as designed. The PPUs were relocated with the radiators, the power management system had to be relocated and augmented, and the xenon feed system had to be modified to facilitate re-fueling of the spacecraft for reuse. Eventually, the structures would only be used as a tank farm, which has to be supported at the base to avoid requalification. This structural configuration presents potential issues when considering design for reuse ${ }^{16}$, specifically accessibility of critical components to facilitate inspection and replacement. The option 2 Hybrid concept is the result of designing a Hybrid concept that uses the ARM EP components rather than structure, and can fit in the Space Launch System (SLS) $8 \mathrm{~m}$ shroud rather than the larger $10 \mathrm{~m}$ shroud. For both options the solar arrays are sized to provide $435 \mathrm{kWe}$ of power at $1 \mathrm{AU}$ at beginning of life (BOL) in order to provide enough power for the vehicle at end of life at Mars distance. The spacecraft is still power limited even at this power level and would benefit from more array power based on trajectory analysis of crew missions. The chemical propulsion system for both concepts uses monomethylhydrazine (MMH) and nitrogen tetroxide (NTO) in a pressure fed system with 10 Aerojet R-42 engines ${ }^{17}$ at nominal $\mathrm{I}_{\mathrm{sp}}$ of 303 seconds that can produce $890 \mathrm{~N}$ of thrust with an oxidizer-tofuel ratio of 1.65. If a Hybrid spacecraft is built, an improved version of the thruster, such as the R-42 DM engine ${ }^{18}$ with the same thrust but an $I_{s p}$ of 327 seconds and an oxidizer-to-fuel ratio of 1.0, would be a significant improvement and reduce the chemical propellant and oxidizer load. In the architecture, the HPS concept is used for both crew and cargo missions to minimize the number of unique elements. Hybrid Option 1 was used for assessment of both crew ${ }^{19}$ and cargo ${ }^{20}$ trajectories for appropriate years with assumed degredation to determine Earth departure dates and propellant loads. Option 1 was chosen, to be conservative, because it is heavier and has a greater propellant load. It is approximately $21.5 \mathrm{t}$ inert with a $23.1 \mathrm{t}$ NTO and MMH capacity an an $18.5 \mathrm{t}$ xenon capacity. It is sized to deliver the EMC in-space habitat concept round trip and is able to deliver significantly more payload in a cargo deployment mode, well in excess of the EMC $18 \mathrm{t}$ payload lander concept.

\section{B. Refueling Tanker}

A refueling tanker concept was developed for the integrated Hybrid architecture based, on a tanker designed by the COMPASS team (Figure 3). The tanker is designed to be launched on an SLS 2B to trans lunar injection (TLI), followed by an LGA and slow transit to LDRO via a BLT. The tanker includes tanks for NTO, MMH, xenon, and gaseous helium for repressurization. It carries pumps, manifolds, and connections for transfer of resources to the HPS via the refueling docking port. It performs the required maneuvers for the transfer to LDRO with a bipropellant reaction control system (RCS) system. The version used for the integrated campaign in this paper is sized to accommodate upto $10.1 \mathrm{t}$ of usable NTO and MMH assuming 3.5\% residuals, and up to $19.6 \mathrm{t}$ xenon assuming 2
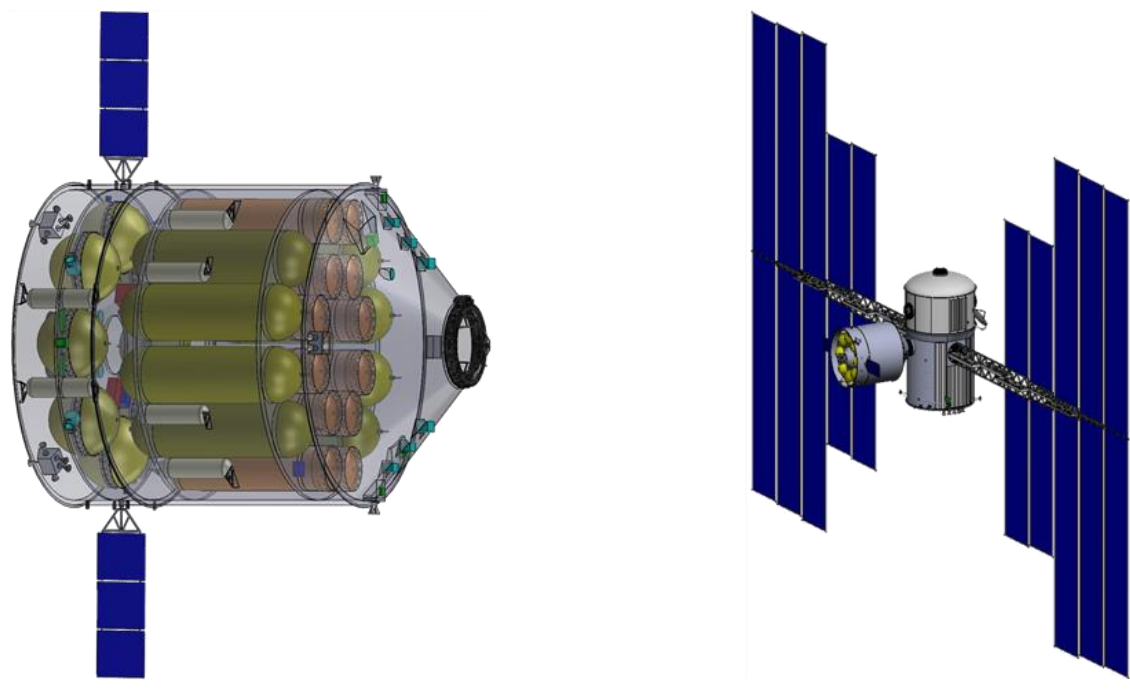

Figure 3. GRC COMPASS Refueling Tanker and Hybrid Propulsion Stage Option 1 
$\%$ residuals. $2 \mathrm{t}$ of the NTO and MMH is used for transit to LDRO; the remaining $8.1 \mathrm{t}$ can be transferred to the client spacecraft. Its estimated inert mass is $5 \mathrm{t}$ for a maximum wet mass of $35 \mathrm{t}$. This is less than the SLS $2 \mathrm{~B}$ performance capability to TLI, so that additional logistics and supplies can be co-manifested on a launch.

\section{EMC Additional Flight Elements}

\section{SLS Block $2 B$}

SLS with exploration upper stage (EUS) and advanced solid boosters, capable of delivering the Orion and $15 \mathrm{t}$ co-manifested payload or $47.5 \mathrm{t}$ of cargo (including payload side adapter assumed to be $2 \%$ ) to trans lunar injection $(\mathrm{TLI})^{21}$ With single shift processing, a single mobile launcher, KSC can nominally launch 2 SLS 2B per year with the ability to launch three in a year, constrained by a maximum rate of no more than five SLS 2B launches in two years.

\section{Orion}

Capable of launch on SLS 2B, transit to and return from cis-lunar space, high or low energy (LDRO, LDHEO) with four crew members.

\section{Deep Space Habitat}

Capable of supporting four crew in space for up to 1200 days, includes storage for logistics and supplies $21.5 \mathrm{t}$ dry and $\sim 20 \mathrm{t}$ logistics and supplies.

\section{Phobos Systems}

Capable of supporting four crew for 500 days on Phobos, includes Phobos habitat, contact and restraint system and pressurized excursion vehicle (PEV) that facilitates longer duration and distance exploration from the Phobos habitat. The Phobos systems are deployed to Mars with a single Hybrid spacecraft prior to the piloted mission.

\section{Taxi}

Habitat and transportation stage capable of supporting four crew for three days maximum, can be resupplied to allow transit between Phobos and 5-sol parking orbit in taxi mode. Taxi is deployed on the same flight with the Phobos systems and is deliverd to in 5-sol orbit prior to Hybrid and Phobos systems transit to Phobos. Is potentially common with the Ascent Stage, but would only include the second stage of the vehicle.

\section{Ascent Stage}

Habitat and 2 stage transportation system capable of supporting 4 crew for 3 days maximum and ascending to 5sol orbit. LOX CH4 propulsion system that is landed with CH4 and LOX is produced from the atmosphere by ISRU system integrated on the descent stage that lands the ascent stage.

\section{Descent Stage}

Can descent from 5-sol orbit to Mars surface with an $18 \mathrm{t}$ payload. Precision landing and hazard avoidance capabilities are required. Gross mass with payload is $43.6 \mathrm{t}$

\section{Mars Surface Systems}

Habitat and surface systems to support human exploration of the surface of Mars. Includes power system, logistics resupply, small pressurized rovers, and offloading equipment at a minimum. Surface systems require four pre-deploy lander missions and one lander for logistics and crew descent for the first crewed mission with a duration of a minimum of 300 days. Each subsequent mission to the Mars outpost requires three landers. An ascent stage, a logistics, and piloted descent vehicle.

\section{Hybrid Campaign Mission Sequence}

This specific example of the Hybrid Architecture was developed with the following ground rules to enable comparison of it with other HAT in-space transportation architecture options:

1) Round trip conjunction class missions to Mars

2) 4 crew travel to destination

3) Aggregate in LDRO 
4) Return transit habitat to LDRO

5) Use SEP systems in development by NASA

6) Use only SLS 2B to launch cargo

7) Use only SLS 2B and Orion to launch crew

8) Deliver Phobos exploration systems to support a 2033 - 2035 Phobos mission

9) Deliver 5 x 18 t payload landers to Mars orbit to support a 2039 - 2042 Mars surface mission

10) Deliver 3 x 18 t payload landers to Mars orbit to support a 2043 - 2046 Mars surface mission

Crew and cargo mission details ${ }^{19,20}$ were developed using the flight elements described in section III and these groundrules. These individual missions have been integrated into an example Mars exploration campaign to identify the total number of transportation elements required and the cadence of resupply needed. The integrated traffic model provides insight into element locaton vs. time and is used to identify potential for reuse of the in-space transportation system.

\section{A. Proving Ground}

The campaign begins with proving ground missions in cis-lunar space, which make up exploration missions (EM) 2-9 of the SLS and Orion (Figure 4). The Proving ground provides time for development of long duration environment control and life support systems (ECLSS), integrated habitation capabilities, dormancy capabilities for human rated systems, as well as refueling and inspection of transportation systems. Each of these capabilities is critical to the first Mars vicinity mission to Phobos. A Phobos precursor mission is also included to prospect potential sites for exploration. A full scale EDL pathfinder mission launching as the Phobos mission starts supports development of EDL and long duration zero boil off liquid oxygen and liquid methane chemical propulsion systems,

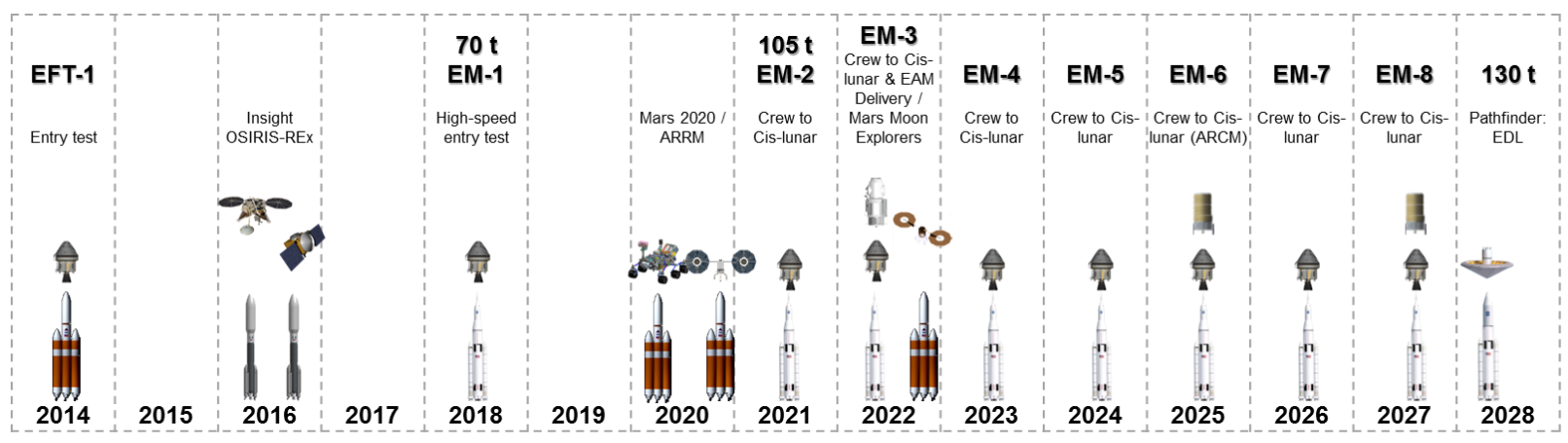

Figure 4. Proving Ground Missions

which are currently used for the landers and ascent stages.

\section{B. Phobos Expedition}

The first piloted expedition to Mars in this campaign is not to the surface of Mars like previous studies; rather the campaign calls for exploration of Mars' moon Phobos first. This delay of Mars surface missions provides additional time for development of the Mars lander and surface systems while exercising the in-space transportation system and supporting a regular cadence of missions. (Figure 5)

\section{Phobos Predeployment Missions}

A long duration surface stay is enabled by pre-deploying the Phobos exploration systems by the first HPS. The HPS is launched pre-integrated with the Phobos Habitat and surface contact system on an SLS 2B and delivers the Phobos habitat to LDRO. In order to maximize injection altitude, and thereby reduce transit time, the HPS is launched with propellant offloaded. Two additional SLS launches then deliver the PEV and Phobos Taxi, each accompanied by a fuel tanker carrying the fuel required by HPS \#1 for the Phobos mission. As this is the first flight of the new habitat and HPS, a crew mission to cis-lunar space will checkout the systems and deliver the final logistics for the Phobos mission. From there the Phobos Cargo stack departs cis-lunar space then performs a BLT and an LGA series to depart the Earth's sphere of influence. The SEP component thrusts during the interplanetary portion of the trajectory to slowly raise the orbital energy to Mars, and the $\mathrm{CP}$ component performs a perigee burn to capture the vehicle into a Mars 5-sol orbit parking orbit. This is a demonstration of the Mars insertion maneuver that the crew will do when they arrive several years later. The Phobos taxi is dropped off in the 5-sol orbit, and the Phobos stack begins a SEP spiral from 5-sol to Phobos. The next payload delivered to cis-lunar space is HPS \#2 
with the crew transit habitat, followed by a launch of another tanker with a logistics module to refuel and resupply the crew transit stack. The crew transit stack then prepares for departure by transferring to LDHEO using a BLT and LGA.

\section{Phobos Crew mission}

The crew launches in an Orion on a SLS 2B to TLI and rendezvous with the stack in LDHEO. Final logistics are transferred from their co-manifested logistics module, after which and the vehicle is ready to depart Earth. The crew then do a phasing maneuver to target one or more LGAs to begin their journey to Mars. Approximately 300-400 days later, after SEP thrusting in high thrust mode (low $\mathrm{I}_{\mathrm{sp}}$ ) during the interplanetary portion of the transit, the crew arrive at Mars via a CP burn at Mars close approach of approximately $250 \mathrm{~km}$ altitude. This burn captures them into a 5-sol orbit where they rendezvous with the predeployed Phobos taxi. The crew checks out the taxi, resupplying it with logistics, and depart from the transit vehicle. The taxi transports the crew to Phobos in less than 3 days, where they rendezvous with the Phobos exploration system. The current nominal concept of operations is to land the Phobos habitat on Phobos prior to crew departure and have the PEV ascend to a rendezvous orbit prior to crew departure from the crew transfer stack. Upon arrival at Phobos, the taxi rendezvous with the PEV, and the crew descend to the Phobos habitat in the PEV. The taxi is left co-orbiting Phobos for the duration of the surface stay.

After the crew successfully arrive at the Phobos habitat, the crew transfer stack begins a "butterfly" maneuver ${ }^{11}$ to reorient the vehicle for Mars departure. This maneuver takes over 200 days and is required specifically for the 2033 Mars opportunity due to the alignment of the arrival and departure orbits at Mars. Other opportunities can be realigned by an apotwist maneuver ${ }^{11}$ that allows the transit vehicle to remain in a 5 -sol orbit for the duration of the crew stay and realign via a plane change maneuver performed at apogee. The crew stay at Phobos for approximately 400 days, where they explore Phobos and teleoperate systems on Mars. When it is time to return to the transit vehicle, the crew ascend in the PEV to the Phobos Taxi, then transfer back to the transit vehicle using similar maneuvers to the transit to Phobos, but in reverse order. After rendezvous with the transit vehicle they transfer all the items to be returned to Earth onto the transit vehicle and leave the taxi in 5-sol orbit. The crew depart Mars and return to Earth in a manner similar to the outbound trip. They use the SEP in high $\mathrm{I}_{\mathrm{sp}}$ mode, since their vehicle is lighter and less thrust is required, to conserve fuel. As they near Earth, an Orion and logistics module are pre-deployed to LDHEO for their return to Earth. This predeployment helps minimize the crew time in space due to launch delays. The Mars transit vehicle then recaptures back at Earth using an LGA series into a LDHEO. The

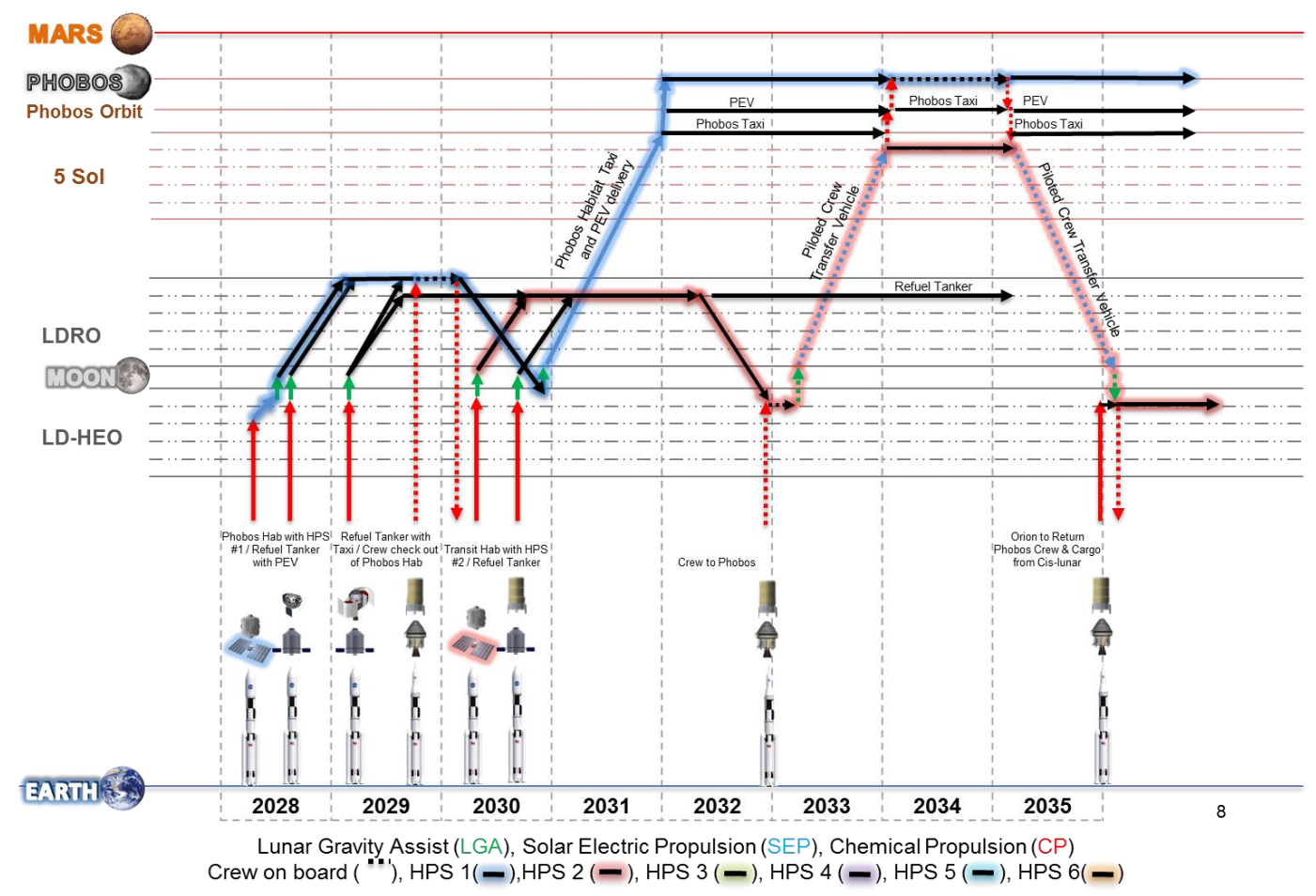

Figure 5. Phobos Expedition Missions

American Institute of Aeronautics and Astronautics 
transfer vehicle and the Orion rendezvous and the crew begin preparations to leave the transfer vehicle. They transfer logistics from the newly arrived module and perform maintenance and safing of the habitat systems prior to dormancy. The crew then load the cargo to be return to Earth in the Orion and depart the transfer vehicle and retune to Earth's surface.

\section{First Mars Surface Expedition}

The first crew to visit the surface of Mars is two opportunities after the Phobos mission and depart Earth in 2039; this is to provide time for deployment of surface systems that enable a full duration stay at Mars on the first visit. Figure 6 represents the launch cadence of elements delivered and provides an approximate representation of the maneuvers performed in order to complete the first Mars surface expedition. Predeployment includes delivery of five landers, three to deliver surface assets, one to deliver the crew ascent stage, and one for crew descent and additional surface assets. The crew mission is the same piloted mission profile flown for the Phobos mission, save for the difference in opportunity.

\section{Predeployment and Preparation Missions}

The 2015 Hybrid campaign includes a full duration Mars surface stay on the first visit requiring five landers to have been deployed prior to the Crew arriving at Mars. The first four landers descend and touch down within one kilometer of each other and lander 5 remains in 5-sol orbit until the crew arrives to rendezvous with the Piloted HPS. For the initial cargo use of HPS they are launched fueled for the mission on an SLS 2B to TLI where they perform an LGA and a BLT to arrive in LDRO approximately 200 days later. Since there are two Mars opportunities for predeployment three landers are delivered on the 2035 opportunity and two landers on the 2037 opportunity. In order to reduce the number of HPS built and launched, the HPS \#2 which delivered the crew to Mars for the Phobos opportunity is used to deliver a lander on the 2037 opportunity. This is possible since HPS \#3, which delivered one of the first set of landers, arrives back at Earth early enough to be used for the first piloted Mars surface expedition. The first tanker flight in 2034 refuels HPS \#2 for lander delivery and the second tanker flight refuels HPS \#3. A crew flies to low energy cis-lunar space (LDRO) to service, re-outfit, and recertify the transfer habitat prior to crew departure in 2039. They stay on the spacecraft while it transfers to high energy cis-lunar space (LDHEO) via a BLT followed by an LGA.

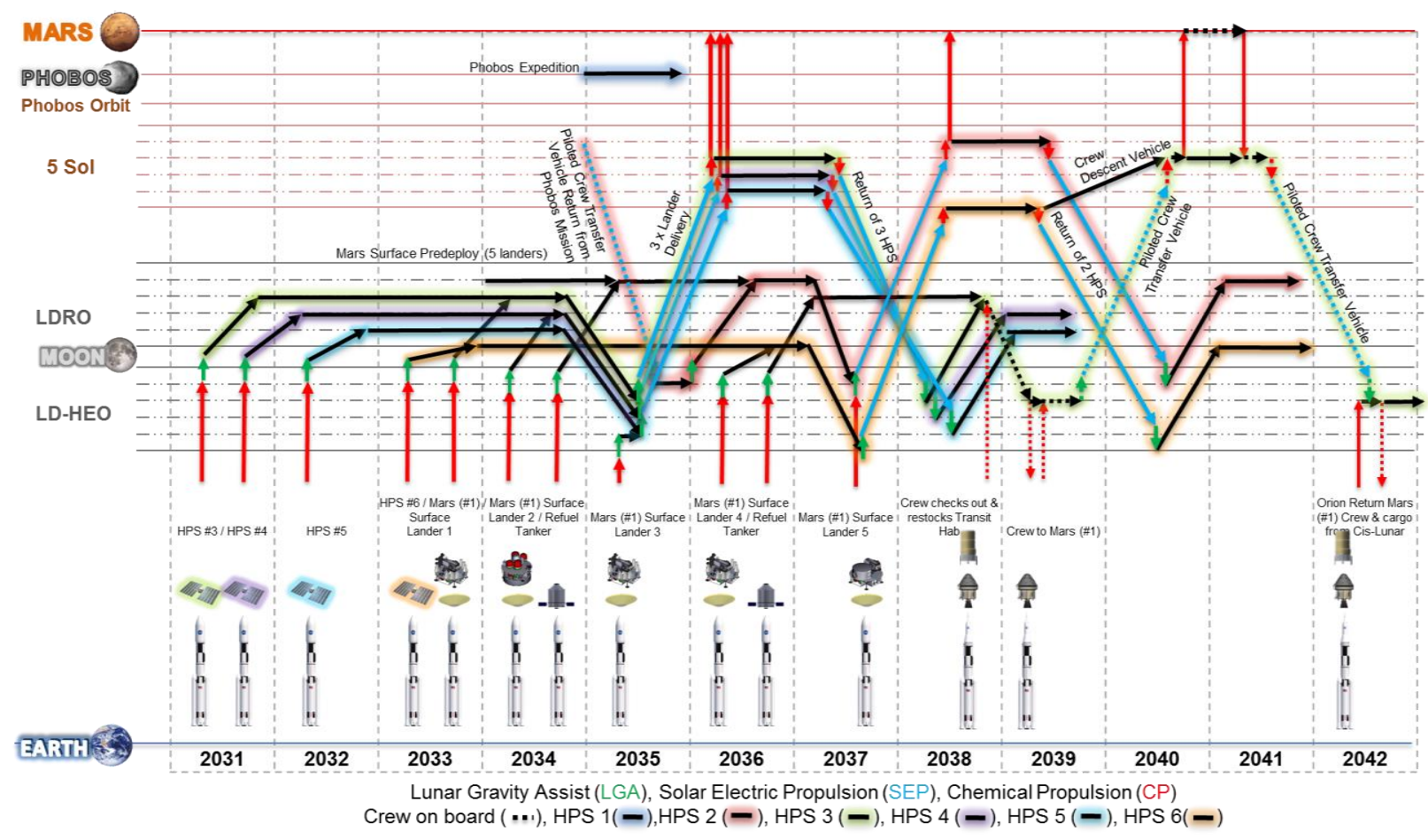

Figure 6. First Mars Surface Expedition Missions 


\section{Piloted Mars Surface Mission}

Once all the surface systems have landed on Mars, the transfer vehicle has been certified, and all systems are functioning nominally, the crew launch on an Orion to rendezvous with the transit vehicle that was moved to LDHEO. Once the crew arrive at the transit vehicle, final logistics are transferred from their co-manifested logistics module, and the vehicle is ready to depart Earth. The crew then do a phasing maneuver and complete an LGA series to begin their journey to Mars. Approximately 400 days later, after SEP thrusting in low $\mathrm{I}_{\text {sp }}$ mode for the majority of the transit, the crew arrive at Mars via a CP burn at Mars close approach of approximately $250 \mathrm{~km}$ altitude. This burn captures them into a 5-sol orbit where they rendezvous with the predeployed Mars lander. The crew land in proximity to the Mars site where the predeployed infrastructure is located. The crew are able to stay on the surface of Mars for nearly 300 days as long as their surface equipment is functioning properly. The transfer vehicle remains in 5-sol orbit, at a predetermined time it performs an apotwist plane change maneuver to realign it for Mars departure. During this time the crew have an opportunity to ascend every 5 days. This abort option is possible due to the availability of an apotwist maneuver to realign the 5-sol orbit for departure in the 2039 opportunity. As the Mars departure window nears, the crew ascend to 5-sol in the pre-deployed ascent stage and rendezvous with the transfer vehicle. After rendezvous with the transit vehicle they transfer all the items to be returned to Earth onto the transit vehicle and leave the ascent stage in 5-sol orbit. The crew depart Mars and return to Earth in the same way as the Phobos mission several years earlier. A CP burn is used for Mars departure, the SEP is used for approximately one year to complete the transfer to Earth where the transit vehicle is recaptured using an LGA series into a LDHEO. The transfer vehicle and the Orion rendezvous and the crew begin preparations to leave the transfer vehicle. They transfer logistics from the newly arrived module and perform maintenance and safing of the habitat systems prior to dormancy. The crew then load the items to be returned to Earth in the Orion and depart the transfer vehicle and return to Earth's surface.

\section{Second Mars Surface Expedition}

The second crew to visit Mars travel to the same surface location and reuse both the surface and in-space transportation infrastructure, represented by Figure 7.

\section{Predeployment and Preparation Missions}

Once the initial reusable Mars surface infrastructure is deployed, only three landers are required for each additional crew mission to the surface: one with an ascent stage, one with additional elements and logistics for the growing outpost, and one for descent with additional logistics. The HPS vehicles needed to transfer these landers to Mars and the crew round trip are already in space. They need to be refueled, rechecked out, and rendezvous with new landers to enable the second Mars surface expedition. Due to phasing constraints associated with the 2041 Earth departure window, the launch of the third lander and refuel tanker \#2 in 2041 are to an LDHEO for rendezvous with the HPS and depart from there via LGA.

\section{Piloted Mars Surface Mission}

The second piloted Mars surface mission has the same concept of operations as the first: the only differences are the $\Delta \mathrm{V}$ splits and time of flight based on the opportunity. For the 2043 opportunity with the reuse of the Hybrid spacecraft, the outbound leg is slightly longer than one year, the maximum surface stay is less than 300 days, and the inbound time of flight is slightly longer than the outbound time of flight. 


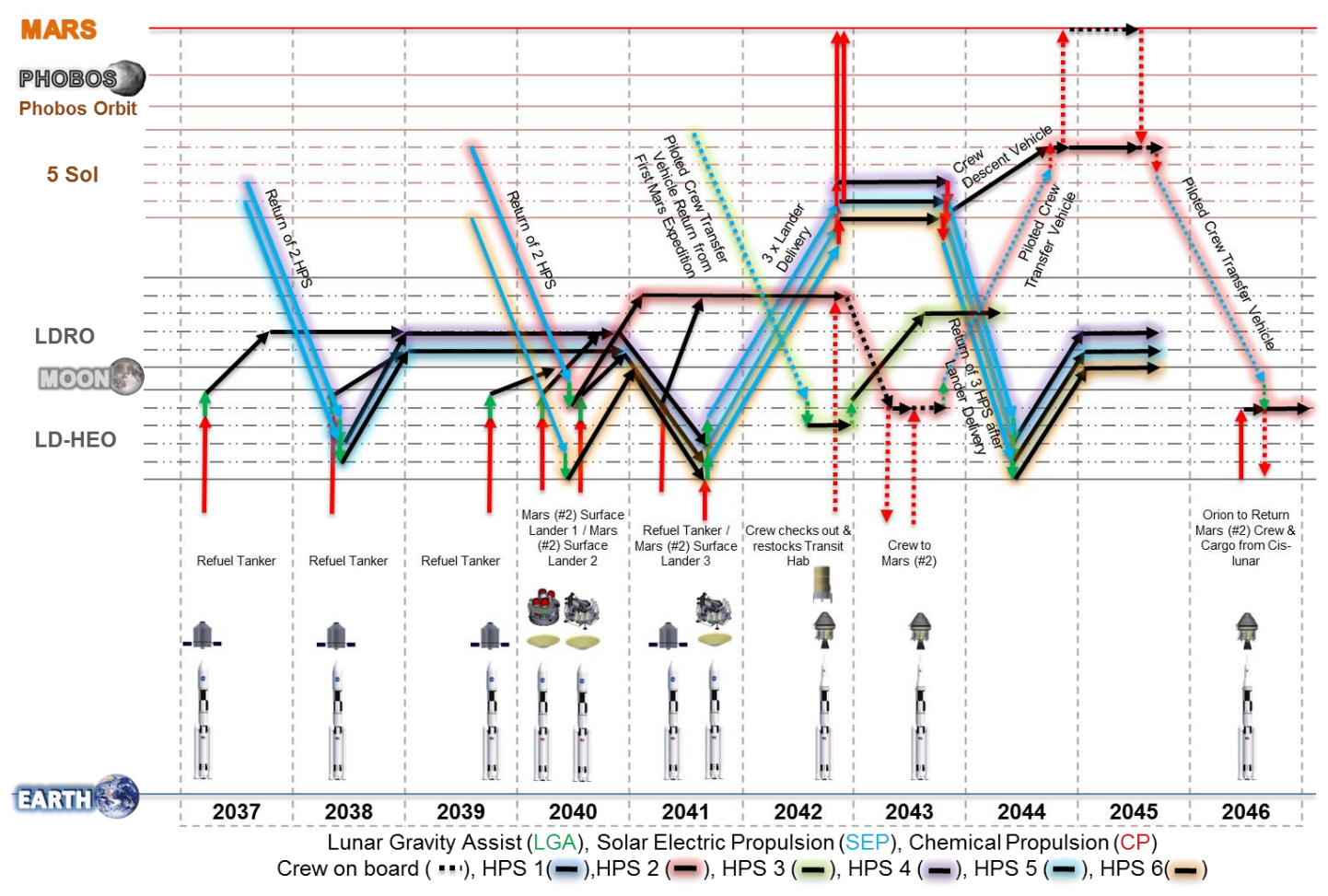

Figure 7. Second Mars Surface Expedition Missions

\section{E. Integrated Manifest and Observations}

Each expedition described individually is part of the larger integrated Mars campaign. Specific launches were manifest to deliver the spacecraft prior to their need date and to balance the SLS lanch cadence which meets the groundrule of two per year and does not require a surge to three per year. Figure 8 depicts the combined launch manifest and traffic model for the three initial expeditions. 32 SLS launches enable three crews of four to visit Mars and four crew rotations checkout and prepare systems for the Expeditions for a total of 28 astronauts launched in an 18 year period. Vehicles travel to Mars every opportunity beginning in 2030 with 11 round-trip Mars transits and one one-way transit performed by the six HPS vehicles. Cargo deployment for the initial mission to Phobos requires half as many launchs as a steady state Mars surface mission (second and beyond) Even with a two opportunity delay all six HPS are launched prior to the first round trip mission with crew in 2033. LDRO is a busy location with as many as four HPS vehicles undergoing checkout and recertification for flight at the peak in 2034. Delivery of five landers prior to the first crew mission causes a surge in activity for the 2035 opportunity where three vehicles travel to Mars at similar times. 


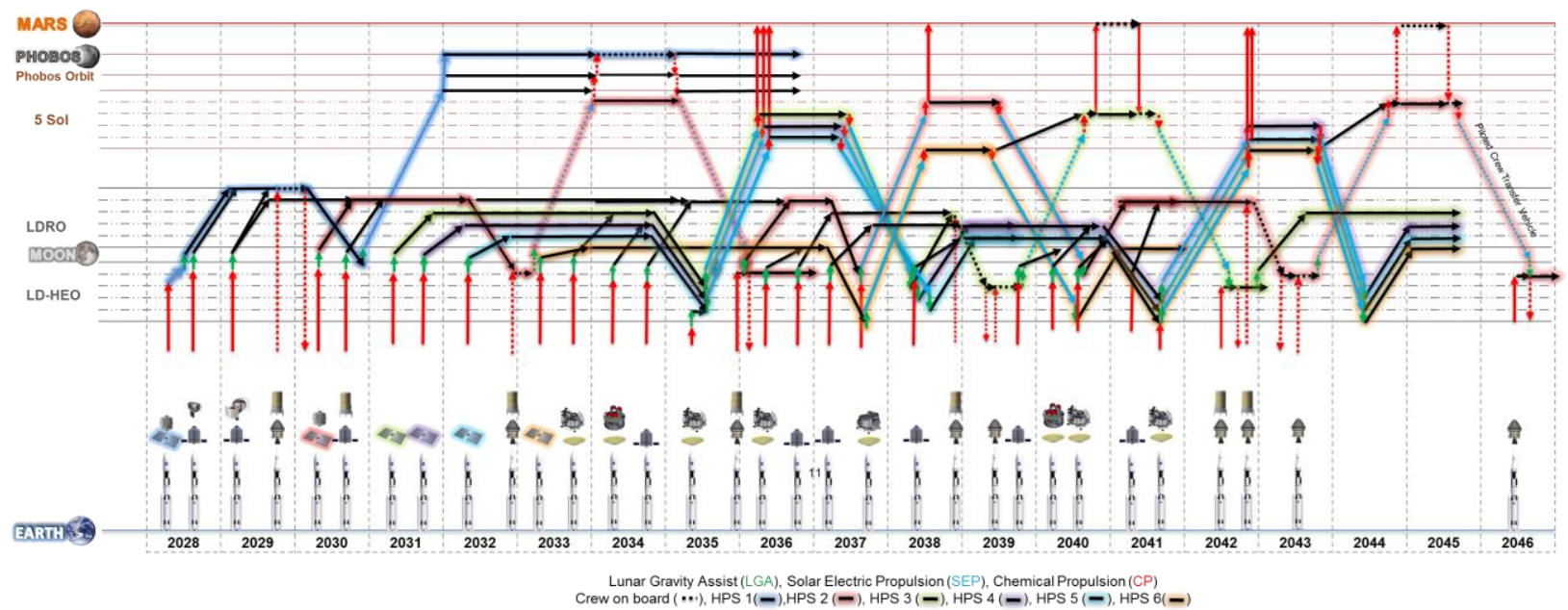

Figure 8. Integrated Campaign for First 3 Mars Expeditions

Figure 9 shows the utilization of each launch, with the green representing unused capability. Launches of the HPS or landers require nearly the full capability of the SLS to TLI. However the 9 refueling launches could be replaced with commercial and international resupply, and the hybrid vehicles (or hybrid with habitat) can be launched offloaded. The launches that are nearly maximizing the capability (1-3 and 9-12) do not require that capability, rather are able to take advantage of it by including logisitics and fuel. Much of the unused capability in the later years are refueling tankers that do not need to deliver logistics or Orion launches that are also not utilizing their comanifested payload capability. Further inspection of each payload shows resilience to element growth except for the Mars landers. The lander concept is currently estimated to weigh $43.6 \mathrm{t}$ for an $18 \mathrm{t}$ payload capability to the surface of Mars, for the integrated Hybrid architecture presented there is only $3 \mathrm{t}$ of additional growth before operations changes must be made to accommodate delivery. There are two options for these operations. Either launch the refueling tanker for lander delivery to Mars to the lower lander insertion orbit, where it boosts the integrated stack to rendezvous with the HPS that delivers it to Mars, or use the HPS that will deliver the lander to Mars to "fetch" the lander from the lower insertion orbit prior to departure to Mars.

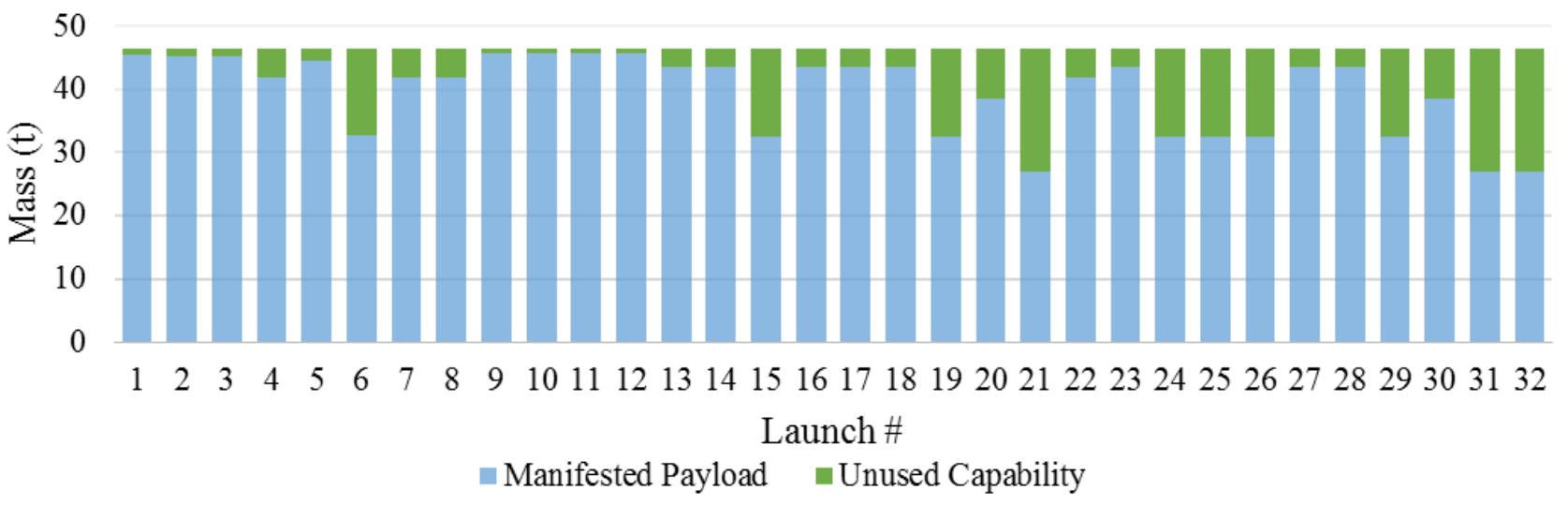

Figure 9. Launch Vehicle Utilization

\section{Conclusion}

This paper has shown the feasibility of the Hybrid transportation architecture to support crew missions to Phobos and Mars in the 2030s and presents an integrated architecture and campaign manifest enabled by reusable Hybrid propulsion stages. A single design for a long duration in-space propulsion stage that combines both CP and EP can be used for both crew and cargo delivery for Mars orbital and suface missions. The HPS vehicles can be reused to 
support multiple missions by flying crew or cargo to Mars every other opportunity. The campaign for the mission dates and destination systems prescribed fills a SLS 2B launch manifest of two fligths per year. Resiliency can be added to the manifest by including commercial and international partner missions to deliver payloads to cis-lunar space. Reuse of the in-space transportation stages provides the opportunity for those missions to deliver fuel, logistics, and other modular equipment to cis-lunar space. Additional refinement of concepts, mission designs, and integrated architectures will continue in order to further illuminate potential pathways on the road to Mars.

\section{References}

${ }^{1}$ Crusan, J., "Evolvable Mars Campaign Report to NASA Advisory Council HEO Committee Meeting," URL: http://www.nasa.gov/sites/default/files/files/20140623-Crusan-NAC-Final.pdf [cited 2 July 2015]

${ }^{2}$ Borowski, S., McCurdy, D.R., and Packard, T.W., "'7-Launch' NTR Space Transportation System for NASA's Mars Design Reference Architecture (DRA) 5.0," 45th AIAA/ASME/SAE/ASEE Joint Propulsion Conference \& Exhibit, Denver, CO., 2009.

${ }^{3}$ Gilland, J.H., et al., "MW-Class Electric Propulsion System Designs for Mars Cargo Transport," AIAA Space 2011 Conference, Long Beach, CA, 2011.

${ }^{4}$ Mercer, C.R., Oleson, S.R., Drake, B., "A Combined Solar Electric and Storable Chemical Propulsion Vehicle for Piloted Mars Missions,” AIAA Space 2013 Conference, San Diego, CA., 2013.

${ }^{5}$ Merrill, R. G., Strange, N., Qu, M., and Hatten, N., "Mars Conjunction Crewed Missions with a Reusable Hybrid Architecture," IEEE Aerospace Conference, March 2015, 2015-8.0104.

${ }^{6}$ Merrill, R.G., Goodliff, K.E., Mazanek, D.D., and Reeves J.D., "Cis-Lunar Basecamp," Global Exploration Conference, March 2012, GLEX -2012.05.5.3x12703.

${ }^{7}$ Parker, J., "Targeting Low-Energy Ballistic Lunar Transfers", American Astronautical Society (AAS) George H. Born

Symposium, Boulder, Colorado. May 13, 2010.

${ }^{8}$ Landau, D., and Longinski, J.., "A Reassessment of Trajectory Options for Human Missions to Mars,” AIAA / AAS

Astrodynamics Specialist Conference and Exhibit, AIAA Washington, DC, 2004.

${ }^{9}$ Landau, D., McElrath, T., Grebow, D., and Strange, N., "Efficient Lunar Gravity Assists for Solar Electric Propulsion

Missions," Paper AAS 12-165, Feb. 2012.

${ }^{10}$ McElrath, et al., "Using Gravity Assists in the Earth-Moon System as a Gateway to the Solar System," Paper GLEX-

2012.05.5.2x12358, May 2012.

${ }^{11}$ Qu, M., Merrill, R.G., Chai, P., and Komar, D. R., "Trajectory Designs for a Mars Hybrid Transportation Architecture,"

AAS/AIAA Astrodynamics Specialist, Vail, CO, 2015 (submitted for publication).

${ }^{12}$ Drake, B., "Human Exploration of Mars Design Reference Architecture 5.0," NASA/SP-2009-566

${ }^{13}$ McGuire, M.L., Oleson, S.R., and Sarver-Verhey, T.R., "Concurrent Mission and Systems Design at NASA Glenn Research Center: The Origins of the COMPASS Team," NASA/TM-2012-217283.

${ }^{14}$ Gerstenmaier, W.H., "Exploration Strategy Update to NAC," URL:

http://www.nasa.gov/sites/default/files/atoms/files/gerst_april_2015.pdf [cited 3 July 2015].

${ }^{15}$ Hofer, R, M.L.Herman, D., Polk, J., Kamhawi, H, and Mikellides, I., "Development Approach and Status of the $12.5 \mathrm{~kW}$ HERMeS Hall Thruster for the Solar Electric Propulsion Technology Demonstration Mission," 34 ${ }^{\text {th }}$ Annual International Electric Propulsion Conference, Kobe-Hyogo, Japan, July 2015, IEPC-2015-186.

${ }^{16}$ Jefferies, S., et al., "Viability of a Reusable In-Space Transportation System," AIAA Space Conference and Exposition, Pasadena, CA, 2015 (submitted for publication).

${ }^{17}$ Stechman, C., "Development and Qualification of a 890 Newton (200 Lbf) Bipropellant Rocket Engine," AIAA 26 ${ }^{\text {th }}$ Joint Propulsion Conference, Orlando, FL, July 1990, AIAA 1990-2055.

${ }^{18}$ Stechman, C., Harper, S., "Performance Improvements in Small Earth Storable Rocket Engines- An Era of Approaching the Theoretical," AIAA 46 ${ }^{\text {th }}$ Joint Propulsion Conference, Nashville, TN, July 2010, AIAA 2010-6448.

${ }^{19}$ Chai, P., Merrill, R.G., and Qu, M., "Mars Hybrid Propulsion System Trajectory Analysis Part I: Crew Missions," AIAA Space Conference and Exposition, Pasadena, CA, 2015 (submitted for publication).

${ }^{20}$ Chai, P., Merrill, R., and Qu, M., "Mars Hybrid Propulsion System Trajectory Analysis Part II: Cargo Missions," AIAA Space Conference and Exposition, Pasadena, CA, 2015 (submitted for publication). 2014

21"Space Launch System (SLS) Program Mission Planner's Guide (MPG) Executive Overview” SLS-MNL-201 August 22, 